\title{
A Rare Case of Imperforate Hymen Presenting with Perineal Pain
}

Zacharis K*, Klimatsouda M, Donoudis C, Valasoulis G and Daponte A

Department of Obstetrics and Gynecology, School of Health Sciences, University of Thessaly, Greece

*Corresponding author: Konstantinos Zacharis, Department of Obstetrics and Gynecology, University of Thessaly, Viopolis, 41500, Larissa, Greece

Received: J anuary 07, 2021; Accepted: J anuary 21, 2021; Published: January 28, 2021

\begin{abstract}
Introduction: Imperforate hymen is a rare local fusion anomaly of the female reproductive tract and diagnosis can be easily missed. Cyclic lower abdominal pain and amenorrhea are common symptoms in cases left untreated after menarche. Perineal pain may be an unusual presenting symptom of imperforate hymen. In order to avert complications related to delayed treatment, hymenotomy is considered effective management.
\end{abstract}

Case Report: We herein describe a case of a 13-year-old adolescent girl with an imperforate hymen presenting with lower abdominal and perineal pain. The girl underwent and hymenotomy and postoperative period was uneventful. Perineal and pelvic pain relieved instantly after surgical treatment and during follow up her menstrual cycles were regular.

Keywords: Imperforate hymen; Hematocolpometra; Adolescence; Perineal pain

\section{Introduction}

The most common congenital anomaly of the female genital tract leading to vaginal outlet obstruction is imperforate hymen. During embryogenesis, a failure in canalization of the caudal segment of the vaginal plate at its juncture with the urogenital sinus leads to imperforate hymen [1]. The incidence is estimated among $0.05-$ $0.1 \%$ of female newborns, although it may occur more frequently [2]. First symptoms may appear during adolescence; hence, the majority of cases pass unnoticed [1]. Cases with prenatal diagnosis via ultrasonography or clinical diagnosis during neonatal period have been also described [3]. Usually, primary amenorrhea is the presenting symptom in most patients. Cyclic pain of the lower abdomen, and hematocolpometra derivative to the accumulated menstrual blood. Atypical signs and symptoms of imperforate hymen have been also reported [4]. We herein present a case of an imperforate hymen as a cause of lower abdominal pain and perineal pain.

\section{Case Presentation}

A 13-year-old premenarchal adolescent girl presented to our outpatient department complaining of perineal pain and lower abdominal pain that started before 36 hours. She reported loss of appetite due to the pelvic pain. Patient described a feeling of heaviness deep within the pelvis and discomfort in the perineal area with a previously normal passage of stools on a daily base. From past medical history, patient reported several episodes of lower abdominal pain gradually worsened in the past six months, which improved without treatment. General examination of the patient revealed a normal phenotype and well developed secondary sexual characteristics (Stage 4 on Tanner scale). On abdominal examination, suprapubic tenderness was elicited and a mass corresponding to the size of a 13 weeks gravid uterus was palpated. A large mass was detected anteriorly by rectal examination. Examination of the external genital organs, showed a bulging hymenal membrane with

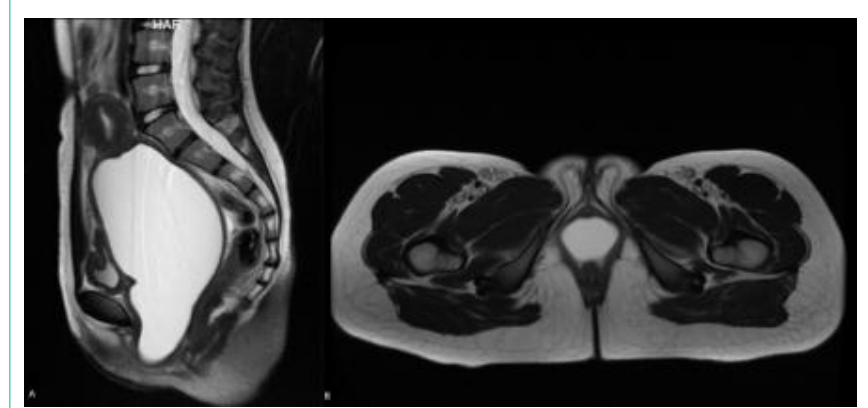

Figure 1: A, Sagittal T2 weighted MRI image showing distention of uterus and vagina by dense fluid (old blood)-Hematocolpometra. B, Axial T2 weighted MRI image showing the lower third of the dilated vagina due to imperforate hymen.

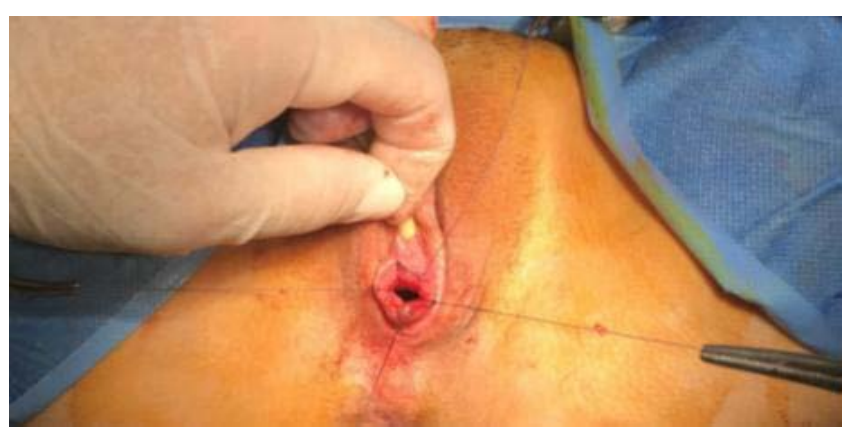

Figure 2: Perioperative finding after hymenotomy with a crucial incision of the hymen, showing the restore of the vaginal outflow.

bluish discoloration when retracting the labia. Patient's, white blood cell count was $13.900 \mathrm{~K} / \mu \mathrm{L}$ (Neutrophils: $78.9 \%$ ), hemoglobin was $11.3 \mathrm{~g} / \mathrm{dL}$ and C-reactive protein was $3.5 \mathrm{mg} / \mathrm{L}$.

Accordingly, patient underwent a transabdominal
Austin J Obstet Gynecol - Volume 8 Issue 1 - 2021

Submit your Manuscript | www.austinpublishinggroup.com

Zacharis et al. (@) All rights are reserved
Citation: Zacharis K, Klimatsouda M, Donoudis C, Valasoulis G and Daponte A. A Rare Case of Imperforate Hymen Presenting with Perineal Pain. Austin J Obstet Gynecol. 2021; 8(1): 1161. 
ultrasonography; Findings included a normal-shaped uterus and the vagina was all filled up with hypoechoic fluid, indicating hematocolpos. Hence, the diagnosis of imperforate hymen was made. A magnetic resonance imaging took place in order to exclude congenital Müllerian anomalies (Figure 1). The patient was brought to the operating theater for surgery under general anesthesia, after obtaining informed consent from her guardian. A hymenotomy with crucial incision of the hymen took place and approximately $500 \mathrm{ml}$ of deep red colored fetid blood evacuated and intravenous antibiotics were administered (Figure 2). The postoperative course of the patient was uneventful and she was discharged on day 2. A follow-up took place six months after treatment. The patient was asymptomatic and she reported regular menstrual cycles.

\section{Discussion}

We hereby report the second case reported in the international literature of a female adolescent who presented with lower abdominal and perineal pain as an atypical presentation of an imperforate hymen [1]. The collection of blood in the vagina and the uterus is the main cause of the majority of clinical presentations of imperforate hymen after menarche. The most frequent symptoms are cyclic pain of the lower abdomen and symptoms of the genito-urinary system $[5,6]$. Data from a recent systematic review on imperforate hymen demonstrated that perineal pain was the presenting clinical genitourinary symptom in $0.4 \%$ of all cases ( 1 in 236 patients) [1].

Diagnosis of imperforate hymen is based on clinical examination of the external genital organs. In addition, a transabdominal ultrasonography may reveal a cystic mass in the pelvis corresponding to the dilated vagina-uterus [2]. These cases require monitoring with magnetic resonance imaging in order to determine the thickness of the imperforate hymen and exclude concomitant congenital Müllerian duct anomalies [7,8]. Delay in the diagnosis and treatment of imperforate hymen may cause complications including infection, endometriosis and infertility consequently [9]. Vaginal agenesis and vaginal septum must be eliminated from differential diagnosis since inaccurate treatment may lead to serious complications. When it comes to female patients with lower abdominal pain and gastrointestinal clinical presentations a multidisciplinary approach consisting of general surgeons and gynecologists is required in order to rule out acute appendicitis which can mimic the signs of an imperforate hymen case [10]. Surgical treatment by hymenotomy with simple vertical, cyclical, cruciate, and T-shaped or X-shaped incisions aims to repair genital tract outflow [11]. Punctures or small incisions without definitive therapeutic management should not be used since they may lead to inoculation of bacteria and cause genital tract infection [12]. The outcome of hymenotomy is considered favorable and reformation after surgery is occasional $[5,11]$.

\section{Conclusion}

Imperforate hymen is an uncommon congenital anomaly of the female genital tract obstruction and diagnosis can be simply achieved. When diagnosis is not confirmed until adolescence, imperforate hymen should be included in the differential diagnosis of girls presenting with pelvic pain and nonspecific symptoms such as perineal pain. Hence, external genital examination should be performed in the beginning evaluation of young female patients complaining for abdominal pain and gastrointestinal or urogenital clinical manifestations. The prognosis of imperforate hymen is favorable, when surgical treatment is performed promptly.

\section{Informed Consent}

Verbal informed consent was obtained from the patient and her guardian for their anonymized information to be published in this article.

\section{References}

1. Lee $\mathrm{KH}$, Hong JS, Jung $\mathrm{HJ}$, Jeong HK, Moon SJ, Park WH, et al. Imperforate Hymen: A Comprehensive Systematic Review. J Clin Med. 2019; 8: E56.

2. Marzuillo P, Guarino S, Apicella A, La Manna A. Imperforate hymen. Turk J Urol. 2017; 43: 102-103.

3. Ayaz UY, Dilli A, Api A. Ultrasonographic diagnosis of congenital hydrometrocolpos in prenatal and newborn period: a case report. Med Ultrason. 2011; 13: 234-236.

4. Ramareddy RS, Kumar A, Alladi A. Imperforate Hymen: Varied Presentation, New Associations, and Management. J Indian Assoc Pediatr Surg. 2017; 22: 207-210.

5. Ossman AME, El-Masry YI, El-Namoury MM, Sarsik SM. Spontaneous Reformation of Imperforate Hymen after Repeated Hymenectomy. J Pediatr Adolesc Gynecol. 2016; 29: e63-e65.

6. Abraham C. Imperforate Hymen Causing Hematocolpos and Urinary Retention. J Emerg Med. 2019; 57: 238-240.

7. Laghzaoui O. Congenital imperforate hymen. BMJ Case Rep. 2016; 2016 bcr2016215124.

8. Kapsalaki E, Daponte A, Deligeoroglou E, Zavos A, Fezoulidis I, Messinis, IE. The role of MRI in young adolescent girls with hematocolpos. Eur J Radiology Extra. 2011; 78: e97-e100

9. Eksioglu AS, Maden HA, Cinar G, Tasci Yildiz Y. Imperforate hymen causing bilateral hydroureteronephrosis in an infant with bicornuate uterus. Case Rep Urol. 2012; 2012: 102683

10. Amponsah-Manu $\mathrm{F}$, Ssentongo $\mathrm{P}$, Arkorful $\mathrm{T}$, Ofosu-Akromah $\mathrm{R}$, Ssentongo A, Hansen-Garshong $S$, et al. Imperforate hymen and leaking hematosalpinx mimicking acute appendicitis: A report of a rare case and a review of literature. Int J Surg Case Rep. 2019; 63: 69-74.

11. Al-Hunaidi O, El-Shazly M, Alkandari M, Ghobashy A, Alhajeri F, Allam A Acute Urine Retention Caused by Hematocolpos After Failed Hymenotomy in 23 Year Old Female. Urol Case Rep. 2015; 3: 50-51.

12. Wong JWH, Siarezi S. The Dangers of Hymenotomy for Imperforate Hymen: A Case of latrogenic Pelvic Inflammatory Disease with Pyosalpinx. J Pediatr Adolesc Gynecol. 2019; 32: 432-435. 